\title{
Da kommt was auf Sie zu
}

\begin{abstract}
Herausforderungen für Berufseinsteiger Während der Einarbeitung erwerben Berufseinsteiger in der Intensivpflege elementares fachbezogenes Wissen und Handlungskompetenzen. Zusätzlich zu den Inhalten der Intensivpflege, -medizin und Medizintechnik gibt es aber weitere Herausforderungen, denen sie sich ebenfalls stellen müssen. Diese Interviewstudie zeigt auf, dass die Auswirkungen der Einarbeitung für die Berufseinsteiger Stress und Belastungen mit sich führen.
\end{abstract}

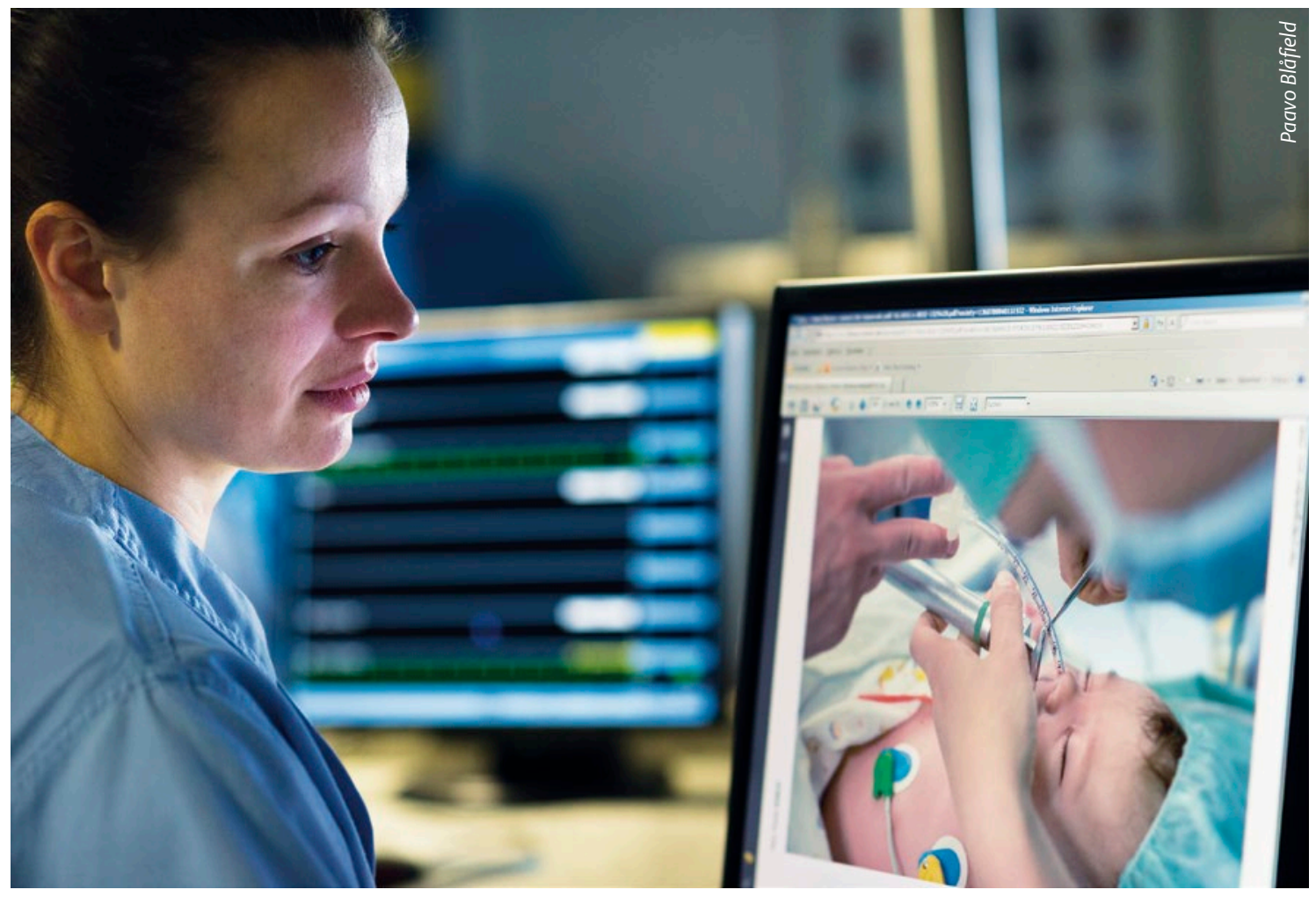

Schaff ich das? Mach ich alles richtig? Akzeptieren mich die anderen? Der Einstieg ins Berufsleben kann für Intensivpflegende sehr beunruhigend sein.

- Birte Vehlow -

Intensivpflegende haben beim Einstieg in den Beruf viele Hürden zu nehmen: Sie stellen sich ihren Befürchtungen vor Beginn der Einarbeitung, müssen mit Berührungsängsten umgehen lernen, die eigene Rolle finden, sich in ein Team integrieren und sehen sich Teamkonflikten ausgesetzt. Sie müssen mit veränderten Rahmenbedingungen und widersprüchlichen Anforderungen umgehen lernen und sich Wissen aneignen, um selbstständig die Arbeit mit den Intensivpatienten zu übernehmen. Eine Einarbeitungskonzeption mit einem festen Einarbeiter, mit langsamem Heranführen an die Thematik und die Patienten, mit Zeit zum Kennenlernen und Üben der neuen Tätigkeiten, Festlegung von erreichbaren Zielen sowie Reflexionen während der Einarbeitung mindern die Ängste und den Stress der neuen Kollegen. Berufseinsteiger werden mit Grenzsituationen in der Arbeit mit Intensivpatienten konfrontiert, denen sie vorher noch nicht begegnet sind. Doch all diese Belastungen werden nicht mit professioneller Unterstützung in der Einarbeitung aufgefangen. Der Einzuarbeitende muss selbst lernen damit umzugehen, bevor die Belastungen eventuell krankhafte Folgen nach sich ziehen. Diesen Aspekten sollte durch spezielle Maßnahmen während der gesamten Einarbeitungszeit mehr Beachtung geschenkt werden, um nach der Einarbeitung motivierte, leis- tungsstarke, selbstsichere und engagierte Kollegen als Teil des Teams zu erhalten.

\section{Einarbeitung von Berufseinsteigern}

In dem für Berufseinsteiger in der Intensivpflege neuen Aufgabengebiet gilt es, bei zunehmender Leistungsverdichtung und einem hohen Grad an Technisierung die komplexe intensivpflegerische Versorgung sowie intensivmedizinische Therapien in kurzer Zeit mit dem Ziel hoher Versorgungsqualität zu erlernen. Eine qualifizierende Einarbeitung bietet den Rahmen für die Vermittlung von elementarem fachspezifischem und praxisbezogenem Wissen sowie den Raum für den Erwerb von Erfahrungen. Dieses dient der Entwicklung, 
Förderung und Stärkung der beruflichen Handlungskompetenzen der Pflegenden. Ziel einer jeden Einarbeitung von neuen Mitarbeitern ist es, nach einem vorgegebenen Zeitraum die routinemäßige Patientenversorgung eigenverantwortlich, selbstständig und fachgerecht durchzuführen.

In dieser qualitativen Studie werden Berufseinsteiger als Gesundheits- und Krankenpfleger direkt nach dem Examen definiert, aber auch als Pflegende, die bereits Berufserfahrungen auf anderen Stationen in einem Krankenhaus sammelten und als fachlicher Anfänger auf einer Intensivstation starteten.

Besonders für Einsteiger ohne Berufserfahrung auf einer Intensivstation ist der Kompetenzerwerb prioritär. Benner beschreibt nach dem Dreyfus-Modell, dass Pflegende ohne jegliche Berufserfahrung noch keine Erkenntnisse über Situationen im Arbeitsgeschehen besitzen, bei denen sie eigenverantwortlich aktiv reagieren müssen. Das bedeutet, sie mussten bisher noch keine Situationen interpretieren und Handlungen ableiten. Sie erlernten bisher, sich anhand von Messwerten oder anderen objektivierbaren Zeichen $\mathrm{zu}$ orientieren und diese zu deuten. Sie nehmen vorerst auch nur einen kleinen Anteil komplexer Situationen wahr, da sie sich auf einzelne Aufgaben konzentrieren. Die vielfältige Gesamtsituation wahrzunehmen und die Werte in den Kontext von anderen Messwerten, Beobachtungen und weiteren Anzeichen zu bringen, setzt die Routine der Basisaufgaben voraus. Außerdem müssen Erfahrungen in dem Zusammenspiel einzelner Geschehnisse gemacht werden. Berufseinsteiger müssen die Kompetenz erlangen, den Patienten und die gesamte Situation $\mathrm{zu}$ überblicken und Prioritäten setzen zu können. ${ }^{1}$

\section{Herausforderungen für \\ die Berufseinsteiger}

Die Ergebnisse dieser Untersuchung belegen eine Belastung für den Berufseinsteiger während der Einarbeitung, die von Unsicherheiten und Ängsten begleitet wird. In der Ergebnisdarstellung kennzeichnen „IB“ und eine fortlaufende Nummer die Zitate der Berufseinsteiger aus den Interviews, „IE“ steht für die Zitate der Einarbeiter. Der Absatz weist auf die Stelle des
Textbeispiels im Transkript hin. Die Zitate sind Einzelaussagen.

\section{Befürchtungen vor Beginn der Einarbeitung}

Bereits vor der Einarbeitung beschrieben die Berufseinsteiger Ängste. Sie sorgten sich, etwas falsch zu machen oder Dinge nicht zu schaffen. „Aber, ja, vielleicht ist es auch ganz gut, dass ich nicht mit dem ganzen Wissen, was hier von mir erwartet wird, hier angefangen hab. Weil, das ist natürlich am Anfang schon ein bisschen beängstigend“ (IB5; Abs. 35). Auch die zeitgleiche Einarbeitung neuer Kollegen hat Einfluss. „Wenn ich dann noch zum Vergleichen jemand gehabt hätte, ich glaube, ich wär untergegangen vom Druck“" (IB7,2; Abs. 17).

\section{Berührungsängste}

Die Patientenklientel ist für die meisten unbekannt und durchaus beunruhigend. So muss sich ein Berufseinsteiger überwinden, „dass man sich mal traut, einen Patienten auch mal anzufassen, der verkabelt ist" (IE1; Abs. 12).

\section{Rollenfindung}

Der Einzuarbeitende muss sich in seine neue Rolle einfinden: „Also es war wirklich bisschen wie so ein Schülerdasein“ (IE3; Abs. 19). „Man (...) denkt, ich habe drei Jahre fast gar nichts gelernt in der Ausbildung“ (IB5; Abs. 35). Einerseits fehlt dem Berufseinsteiger das nötige Wissen für diesen Fachbereich, und während der Einarbeitung muss viel zugeschaut und gelernt werden, andererseits ist er aber ausgelernter Pflegender mit eigener Durchführungsverantwortung. Unzufriedenheit mit sich selbst kommt bei den Pflegenden mit Berufserfahrung auf: „Als ich hier angefangen hab, hatte ich das Gefühl, ich weiß gar nichts“ (IB2; Abs. 34).

Außerdem sind Mitdenken und das Einbringen in die Pflege und Therapie auf den Intensivstationen erwünscht. Diese $\mathrm{Zu}-$ sammenarbeit zwischen Medizinern und Pflegenden muss ebenfalls erlernt werden.

\section{Integration in das Team}

„Es ist einfach schwierig, (...) in diesem Bereich halt wirklich Fuß zu fassen, und da ist die Einarbeitung für mich einfach elementar“ (IE1,1; Abs. 31). Das bezieht sich sowohl auf die fachlichen als auch auf die zwischenmenschlichen Komponenten. „Also, das war so der Schwerpunkt, im Team dann irgendwo anzukommen, ohne jetzt zwischen die Fronten zu geraten, war wirklich schwierig. So, und ja, als Neuer und frisch Examinierter ist man natürlich dann auch immer noch mal ein bisschen, ja, belächelt“ (IB7,1; Abs. 37). Die Integration in ein bestehendes Team, die Anerkennung bei den Ärzten und die Rollenfindung spielen eine beachtliche Rolle. „Gewissermaßen muss man sich ja halt einen Namen machen, beziehungsweise ein Standing hier aufbauen“(IE1,1; Abs. 47). „Es gibt die Cliquen, und die sind cool, die sind nicht so cool, wo ordnet sich dieser Mensch jetzt ein“ (IB7,1; Abs. 37). So geraten Neulinge ungewollt durchaus auch in Teamkonflikte und müssen lernen damit umzugehen. „Ich hab die Bemerkungen schon durchaus wahrgenommen, dass die einen die anderen schlechter finden und dass ich ja jetzt von einem eingearbeitet werde, der schlechter ist sozusagen“ (IB7,1; Abs. 37).

\section{Veränderte Rahmenbedingungen}

Erschwerend kommt auch der Wohnortwechsel dazu, erzählte ein Befragter, der für die neue Arbeitsstelle nach Hamburg gezogen war. Familie und Freunde waren zur mentalen Unterstützung nicht mehr in der Nähe, und parallel zur Einarbeitung musste er sich an die neue Wohnumgebung gewöhnen. Andere Rahmenbedingungen, Strukturen und Abläufe in der neuen Klinik müssen akzeptiert oder es muss ein Anstoß zur Veränderung gegeben werden. „Das war irgendwie so schade, ich kannte das aus meinem Haus, zack, stand der Neurologe schon auf der Schwelle, (...) dass dann erst mal einer angerufen werden muss, zum Konsil bestellt werden muss (...)“ (IB7,2; Abs. 7). Diese Situation hat der Befragte als unbefriedigend erlebt.

\section{Widersprüchliche Anforderungen}

Auf widersprüchliche Anforderungen trifft der Einzuarbeitende oft, wenn er von anderen Kollegen als vom zugeteilten Mentor in der Praxis begleitet wird. „Was ja das größte Problem immer ist: Jeder macht das ja nach bestem Wissen und Gewissen“ (IE1,1; Abs. 11). Unterschiedliche Hand- 
lungsabläufe durch fehlende Standards und Verfahrensanweisungen oder das Abweichen von den Vorgaben erschweren den neuen Kollegen das Lernen. Sie können noch nicht zwischen korrekten und eher ungenauen Durchführungen differenzieren. Der Einzuarbeitende muss sich den Eigenarten und den Schwerpunkten der Anleiter anpassen. Bei wechselnden Mentoren muss er jene erst neu von seinen Kompetenzen überzeugen, um dann wieder in die eigentliche Übungs- und Lernphase einzusteigen.

Dazu kommen der Umgang und die Motivation untereinander. „Ich bin auch schon mit Kollegen mitgelaufen (...), wo man dann auch gemerkt hat, (...) ist jetzt nicht deren Lieblingsbeschäftigung“ (IB2; Abs. 65). Der Befragte fühlte sich „wie ein Klotz am Bein“ (IB2; Abs. 67) und setzte sich dann unter Druck, um dem Kollegen nicht so viel Arbeit zu bereiten.

\section{Aneignung von Wissen}

Zum Anfang „hab ich auch noch viel gelesen, nebenbei, und dann war ich nachher ziemlich überlagert mit all den Infos (...), die da auf mich zukamen, und mit all den Eindrücken. Da konnte ich dann parallel gar nichts mehr zu lesen und lernen. Das ging gar nicht mehr in meinen Kopf rein“ (IB9; Abs. 40). Von dem Berufseinsteiger wird eine hohe Eigeninitiative bezogen auf das Erfragen und Erlesen von Hintergrundwissen und Zusammenhängen erwartet. Aufgrund der Menge des Lernpensums muss das in der Freizeit erledigt werden. „Also, ich fand die Menge, was ich eigentlich lernen sollte in dem Zeitraum, zu viel. Also, grade so, was spezielle Krankheitsbilder angeht und sowas (...). Da hätte man wirklich Doppelschichten schieben müssen, zu Hause noch“ (IB2; Abs. 23). Ein Berufseinsteiger berichtete: „Aber am Ende der sechs Wochen [= am Ende der Einarbeitungszeit - Anm.d. Autorin] hab ich dann das Gefühl gehabt, so, ok, jetzt kannst du starten, auch wenn du ganz viele Sachen noch nicht weißt“" (IB10; Abs. 31).

\section{Ende der Begleitung \\ durch den Einarbeiter}

$\mathrm{Zu}$ der Freude auf das alleinige Arbeiten gesellt sich die Eigenverantwortung. „Ich hab eigentlich die Zeit nach der Einarbeitung als am schlimmsten empfunden. So einfach, so, verantwortungstechnisch“ (IB9; Abs. 40). Der neue Kollege arbeitet dann eigenverantwortlich und es ist nur noch ein Ansprechpartner vorhanden. „Und so nach acht Monaten oder so fängt dann an der Druck zu sinken. Und man bleibt (...) viel mehr entspannt. Irgendwie, der Stresspegel ist einfach nicht mehr konstant so hoch“ (IB9; Abs. 40). Ein Einarbeiter weist auf die Gefahr des Burn-outs hin. Dieser Stress und Druck beeinflusst die psychische Gesundheit. Der Stress zeigt sich durchaus auch physisch in Form von Schweißperlen auf der Stirn. Zur Kompensation trieb ein Berufseinsteiger Sport. „Ich hab extrem viel Sport gemacht. Weil ich einfach echt einen hohen Stresslevel hatte, und dann hab ich einfach die Erfahrung gemacht, ich bin abends ins Bett gegangen und ,ticker ticker‘, mein Kopf hat nicht abgeschaltet“ (IB9; Abs. 62). Es ist „wahnsinnig viel Verantwortung“ (IB5; Abs. 13). Zusätzlich belastet der unterschiedlich hohe Arbeitsaufwand. Der eigene Anspruch, alle Arbeit zu erledigen, ist groß - vor allem, weil der Berufseinsteiger in der Einarbeitungszeit beigebracht bekommt, sich an einen strukturierten Ablauf zu halten und alles zu schaffen. „In der Einarbeitung geht man ja schon so danach, bei den (...) Patienten auch alles immer wirklich zu schaffen. Einfach, weil ich auch Zeitmanagement üben musste“ (IB9; Abs. 50). „Da haben auch sehr viele mit zu kämpfen, so dieses, oh, ich habe meine Arbeit nicht geschafft. Da muss man auch sagen, ja, die konntest du auch nicht schaffen. Ich hätte die auch nicht geschafft. Also das ist dann so“ (IE4,2; Abs. 35). Das zeigt die Wichtigkeit des Erlernens der Prioritätensetzung in der Intensivpflege und -medizin.

\section{Auswirkungen auf \\ die Berufseinsteiger \\ Entwicklungsunterstützung}

Der Stresspegel eines Befragten am allerersten Tag regulierte sich durch das Mitlaufen neben dem Mentor schnell. „Ich hatte einen extrem positiven Eindruck, sodass irgendwie innerhalb von $20 \mathrm{Mi}-$ nuten mein Stresslevel extrem gesunken ist, irgendwie so, dass ich einfach wusste, so, ich steh jetzt heute wirklich nur so dabei. Ich guck mir das jetzt alles nur an und, weil ich wirklich das Gefühl hatte, ich darf (...) einfach schnuppern und gucken, ob mir das gefällt" (IB9; Abs. 60). Das vorsichtige Heranführen an den neuen Arbeitsbereich nimmt die Angst. In den ersten Tagen der Einarbeitung kommt viel auf den Berufseinsteiger zu. „Ein nicht Erfahrener (...), die sind erstmal (...) völlig begeistert (...) und sind aber auch nach vier Tagen komplett erschlagen, von der Masse, die an Eindrücken und Information auf die einschlägt“ (IE11; Abs. 36). „Die Informationen kommen alle so richtig heftig auf einen zu“ (IB7,1; Abs. 25). Strukturierte Einarbeitungsinhalte und festgelegte Etappenziele können den Stress vermindern. „Es ist auch ganz nett, wenn man sich das so durchliest, was in der ersten Woche von einem verlangt wird, dann denkt man, okay, das kriege ich (...) hin, das ist alles nicht so schlimm. Das (...) wird nicht so hoch gesteckt“ (IB9; Abs. 13).

\section{Vermittlung von Sicherheit}

„In der Einarbeitung, in der Hälfte [= nach ca. sechs Wochen - Anm.d. Autorin], würde ich sagen, war der Stresspegel sehr gering, weil es wirklich ein sutscher Einstieg ist, somit, da wird schon drauf geachtet. Und man hat einfach immer einen so hinter sich“ (IB9; Abs. 40). Unterstützend in der Einarbeitungszeit wirken Gespräche zur Reflexion. Dem Einzuarbeitenden wird aufgezeigt, was er schon kann und was er noch lernen muss. „Die Gespräche, die dann über das halbe Jahr am Anfang geführt wurden, haben auch schon dazu beigetragen, denke ich, dass man darüber überhaupt erst mal nachgedacht hat, was man für eine Entwicklung gemacht hat in der Zeit" (IB7,2; Abs. 15). Das bringt ein befriedigendes Gefühl mit sich. Die praktische Begleitung in der Einarbeitungszeit und der Ansprechpartner in der Phase danach vermitteln Sicherheit. Außerdem liegt die Verantwortung in der Einarbeitungszeit bei dem Mentor.

Die Qualität der Einarbeitung spielt auch eine bedeutende Rolle. „Je besser sie eingearbeitet sind, desto sicherer fühlen sie sich“ (IE6; Abs. 35). Auf einer Intensivstation ist man nie allein, es ist immer ein Kollege oder ein Arzt in Rufweite, das ist beruhigend. Unterstützungsangebote 
von Kollegen in der Eingewöhnungsphase helfen an Tagen mit hohem Arbeitsaufkommen. Das Erlangen von Routine in den täglichen Handlungen und Abläufen durch praktisches Üben führt ebenfalls zu mehr Sicherheit.

\section{Eigenes Ethik- und Pflegeverständnis}

Ein Befragter berichtete von einer Stresssituation, die ausgelöst wurde durch die Lebensbedrohung des zu betreuenden Patienten. Diese Situation brachte ihn nach Stabilisierung des Patienten zum Weinen. Solche Grenzsituation kennen auch andere Kollegen. Das Team stieg in diese Thematik ein, die Teammitglieder berichteten von ihren belastenden und beanspruchenden Erfahrungen. „Es ist ein junger Mensch, der fängt erst an. Damit kann der im Grunde genommen (...) so viel anrichten, dass er sagt, ich kann den Beruf nicht machen. Ich habe mir das ganz anders vorgestellt“ (IE4,2; Abs. 47). Die Einzuarbeitenden müssen ihr Pflegeverständnis und ihr Ethikempfinden in der Anfangszeit definieren. Sie müssen für sich eine gesunde Distanz zur Arbeit aufbauen und sich damit auseinandersetzen, ob dieser Job die Arbeit beinhaltet, die sie zukünftig gern übernehmen möchten.

\section{Arbeitszufriedenheit}

Die Einarbeitung steigert die Wissbegier der Berufseinsteiger und steckt erreichbare Ziele, deren Erreichung zufrieden macht. Aber die Arbeitszufriedenheit entwickelt sich erst nach der Einarbeitung, wenn der Berufseinsteiger reflektieren

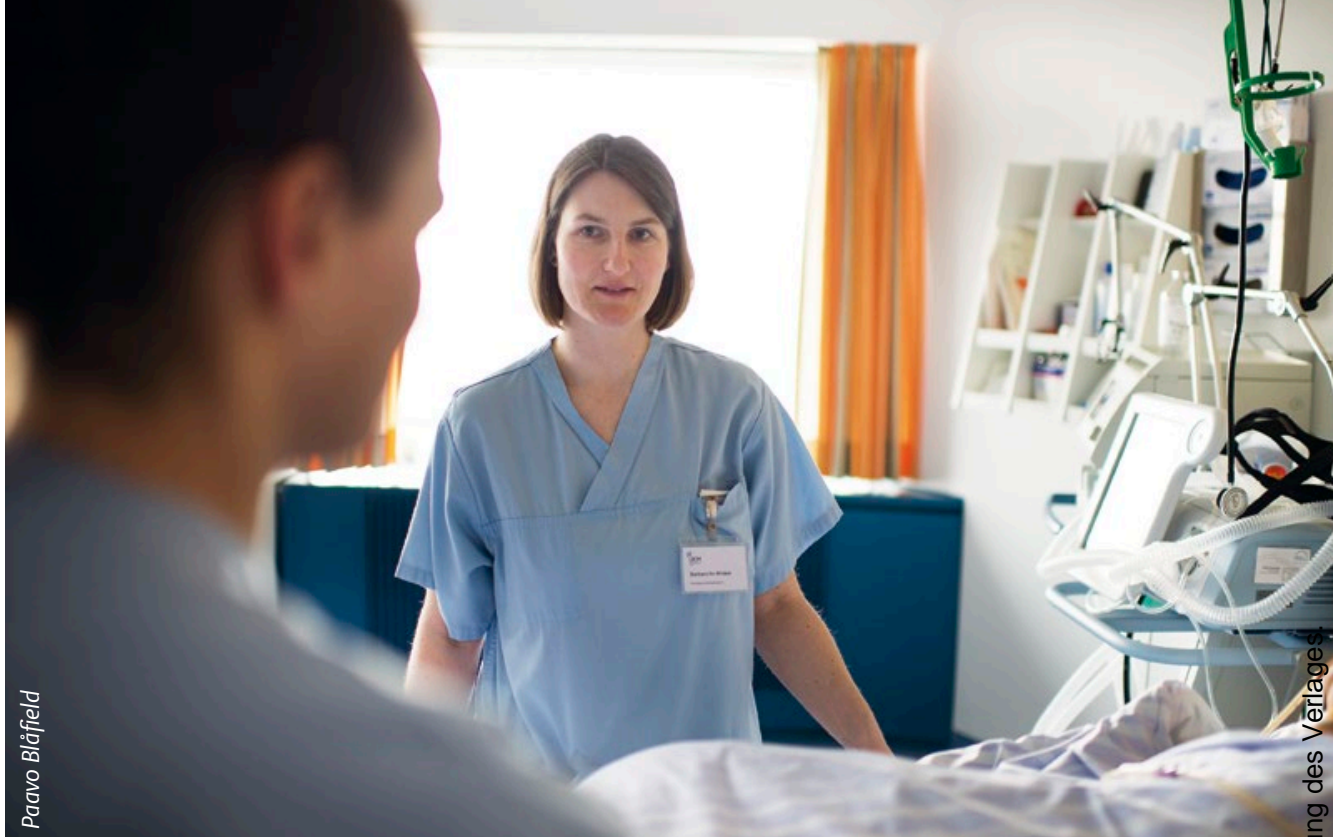

Die Einarbeitung findet oft zusätzlich zum Praxisalltag statt - was zu einer höheren Belastung der Einarbeiter führt. kann, ob ihm die Arbeit liegt. Einen großen Einfluss auf die Arbeitssituation und -zufriedenheit hat die Integration in das Team. Die Einarbeitung ist Teil der fachlichen und persönlichen Entwicklung der Einzuarbeitenden.

\section{Herausforderungen \\ für die Einarbeiter}

Den Einarbeitern fehlt es an Zeit, die Einarbeitung zu planen und vorzubereiten, dieses machen sie dann zusätzlich zum Pflegealltag. Durch das aktuelle und vollständige Fach- und Organisationswissen der Einarbeiter stehen sie häufig den anderen Kollegen für Fragen zur Verfügung. „Also, wenn es irgendwelche fachlichen Fragen gibt, dann kommen die meisten zu mir. Also ich werde ständig irgendetwas gefragt“ (IE4; Abs. 31). Viele durchzuführende Einarbeitungen in kurzen Zeiträumen, fehlende Einarbeitungs-
Tab. 1 Spezifizierung der Stichprobe.

\begin{tabular}{|c|c|c|c|c|c|c|}
\hline \multicolumn{7}{|l|}{ Berufseinsteiger } \\
\hline Alter in Jahren* & 23 & 29 & 32 & 27 & 27 & \\
\hline Geschlecht & w & w & w & w & w & \\
\hline Anzahl der Berufsjahre in der Pflege* & 1 & 3 & 4 & 5 & 6 & \\
\hline \multicolumn{7}{|l|}{ Einarbeitende } \\
\hline Alter in Jahren* & 30 & 35 & 44 & 42 & 46 & 51 \\
\hline Geschlecht & w & $\mathrm{m}$ & $\mathrm{m}$ & w & w & $\mathrm{m}$ \\
\hline Anzahl der Berufsjahre auf der Intensivstation* & 10 & 11 & 19 & 21 & 21 & 24 \\
\hline Anzahl der Berufsjahre in der Pflege* & 10 & 14 & 20 & 22 & 24 & 24 \\
\hline
\end{tabular}

* Zum Zeitpunkt der Befragung richtlinien und Pflegestandards und die Verantwortung für Berufseinsteiger und deren Handlungen führen zu Belastungen. Personalengpässe beeinflussen die Durchführung der Einarbeitung negativ. Es kommt zur Überforderung der Einarbeiter, wenn mehr Patienten versorgt werden müssen als aufgrund der Einarbeitungsituation vorgesehen sind. Die Durchführung einer geplanten Einarbeitung auf einer Intensivstation gestaltet sich manchmal schwierig. „Man kann natürlich nicht immer (...) über A nach B nach $C$ gehen, sondern man muss auch manchmal hin und her springen" (IE4,1; Abs. 8). Das ist abhängig vom einzelnen Arbeitstag, da Intensivpflege sehr inkonstant sein kann. Außerdem werden spezielle Therapieformen nicht täglich und bei allen Patienten eingesetzt, was bei der Patientenauswahl für die Einarbeitung eine Rolle spielt. All das erfordert eine hohe Flexibilität der Einarbeiter.

\section{Methode}

Ziel dieser Untersuchung war es aufzuzeigen, welchen Herausforderungen sich Berufseinsteiger und Einarbeiter auf einer Intensivstation neben dem Erlernen der Fachlichkeit stellen müssen. Der Nutzen zeigt sich in der Reflexionsmöglichkeit der Einarbeitungssituation und gegebenenfalls einer Anpassung und Schwerpunktverlagerung für die Einarbeitungsstrategie.

Das gewählte Studiendesign ist eine qualitative Befragung einer Gelegenheitsstichprobe mittels Leitfadeneinzelinterviews. Qualitative Forschung eignet sich besonders zur Felderkundung und kann 
als Basis oder Ergänzung von quantitativen Studien dienen. ${ }^{2}$ Der Vorteil von Interviews liegt in der breiten Antwortvielfalt und der großen Fülle von Informationen, die damit generiert werden können. ${ }^{3}$ Die Auswertung der Transkripte erfolgte mittels der inhaltlichen Strukturierung der qualitativen Inhaltsanalyse von P. Mayring. Das angewendete Kategoriensystem wurde aufgrund der Leitfragen generiert und die Unterkategorien aus den Nachfragen gebildet. ${ }^{4}$
Die zugrundeliegende Fragestellung lautete: Welche Auswirkungen hat die Einarbeitung von intensivunerfahrenen Gesundheits- und Krankenpflegern mit und ohne Berufserfahrung in die Intensivpflege auf die Mitarbeiter?

Die Interviews wurden mit Gesundheits- und Krankenpflegern geführt, die 6-16 Monate zuvor eingearbeitet worden waren. Die Befragten besaßen vorher keinerlei Berufserfahrungen in der Intensivpflege. Zusätzlich wurden Gesundheits- und Krankenpfleger, die Einarbeitungen auf Intensivstationen durchführen, interviewt.

\section{Stichprobe}

Fünf Interviews mit Berufseinsteigern sowie sechs Interviews mit Einarbeitenden wurden von der Autorin persönlich Face-to-Face durchgeführt. Die Dauer der Interviews mit Mitarbeitern von fünf verschiedenen Hamburger Krankenhäusern betrug zwischen 18 und 48 Minuten. Die Stichprobe wird in $\rightarrow$ Tab. 1 verdeutlicht.

Fazit

Die Einarbeitung von Berufseinsteigern in die Intensivpflege und -medizin ist sehr komplex, umfangreich und durchaus auch belastend. Mitarbeiter, die auf einer Intensivstation beginnen zu arbeiten, müssen sich zusätzlich zum Kennenlernen des Unternehmens und der Räumlichkeiten, der Standards und der Gepflogenheiten sowie der Kollegen besonderen Herausforderungen stellen.

Die Berufseinsteiger müssen ihre Rolle als examinierte Pflegende, aber auch als Einzuarbeitende in einem völlig neuen Berufsfeld definieren. Parallel dazu erfolgt die Integration in das Team. Sie bauen Berührungsängste ab, überprüfen ihre Belastungsgrenzen, ihren Umgang mit Stress und müssen Bewältigungsstrategien entwickeln. Trotz unterschiedlicher Arbeitsweisen der Praxisanleiter und zum Teil fehlender Standards müssen sie ihre eigenen Handlungskompetenzen festigen. Die Besonderheiten in der Intensivpflege führen zum Überdenken des eigenen Pflege- und Ethikverständnisses. „Komplexe klinische Maßnahmen und Bewertungen lassen sich nicht mehr nur den Ärzten allein zuordnen“. 5 Deshalb müssen die Berufseinsteiger unbedingt nicht nur Handlungsabläufe erlernen, sondern sich auch sehr viel Wissen aneignen und Zusammenhänge erkennen. Zum Lernen verwenden die Einzuarbeitenden ihre Frei- und Erholungszeit. Den kognitiven Verarbeitungsprozessen wird somit gar nicht genügend Zeit eingeräumt. Diese Aspekte sollten in der Einarbeitung Beachtung finden und die Berufseinsteiger durch Betreuung und Kompetenztraining gestärkt werden. Eine Integration der Themenbereiche „Umgang mit Stress“ und „Selbstreflexion“ sowie Beratungs- und Coaching-Angebote in der Eingewöhnungsphase neuer Kollegen sind zu überdenken.

Die Ergebnisse dieser Untersuchung können ein Anstoß sein, die eigene Einarbeitungskonzeption zu reflektieren, um gegebenenfalls neue Aspekte aufzugreifen und weitere Maßnahmen aufzunehmen. Die beschriebenen Auswirkungen auf Berufseinsteiger unterstreichen die Notwendigkeit einer qualitativen und strukturierten Einarbeitung. Eine zufriedenstellende Einführung in den Arbeitsbereich dient zusätzlich der Mitarbeitergewinnung durch mündliche Empfehlungen, zudem wird die Bindung des Mitarbeiters unterstützt. Der Fachkräftemangel in der Pflege sowie das Fortschreiten der demografischen Entwicklung und deren Folgen erfordern spezielle Maßnahmen, um kompetente und motivierte Mitarbeiter für ein Unternehmen zu gewinnen und sie daran zu binden. Eine qualifizierende Einarbeitung mit einer psychosozialen Betreuung der neuen Kollegen zur Bewältigung der Herausforderungen ist dabei eine unterstützende Komponente.

Literatur

1 Benner P, Wengenroth M, Staudacher D. Stufen zur Pflegekompetenz: From novice to expert. 2., vollst. überarb. und erg. Aufl. Bern: Huber; 2012

2 Flick U, v. Kardorff E, Steinke I (Hrsg.). Qualitative Forschung: Ein Handbuch (Orig.-Ausg., 10. Aufl.). Reinbek bei Hamburg: Rowohlt; 2013

3 Hopf C. Befragungsverfahren. In: Flick U, v. Kardorff E, Keupp H (Hrsg.). Grundlagen Psychologie. Handbuch qualitative Sozialforschung. Grundlagen, Konzepte, Methoden und Anwendungen. 2. Aufl. Weinheim: Beltz; 1995: S. 177-182

4 Mayring P. Qualitative Inhaltsanalyse: Grundlagen und Techniken. 12., überarb. Aufl. Weinheim: Beltz; 2015

5 Isfort M, Weidner F, Gehlen D. Pflege-Thermometer 2012: Eine bundesweite Befragung von
Leitungskräften zur Situation der Pflege und Patientenversorgung auf Intensivstationen im Krankenhaus. Deutsches Institut für angewandte Pflegeforschung e. V. (Hrsg.), 2012. Online unter www.dip.de/fileadmin/data/pdf/projekte/Pflege_ Thermometer_2012.pdf, letzter Zugriff 22.08.2016

\section{Danke}

Mein Dank gilt Herrn Prof. Dr. med. A. Nienhaus, $\mathrm{MPH}$, Berufsgenossenschaft für Gesundheitsdienst und Wohlfahrtspflege, und Frau Dr.P.H. A. Schablon, Universitätsklinikum Hamburg Eppendorf, für die Unterstützung bei der Studie für meine Bachelorthesis, die diesem Beitrag zugrunde liegt.

\section{Autorin}

Birte Vehlow, B. A.

Fachgesundheits- und Krankenpflegerin für Intensivpflege und Anästhesie. Arbeitet seit ihrem Studium in Pflegeentwicklung und Management als Assistentin der Oberin in der DRK-Schwesternschaft Ostpreußen e.V.

E-Mail: bvehlow@email.de

\section{Bibliografie}

DOI 10.1055/s-0042-118947

Intensiv 2017; 25: 20-24

(c) Georg Thieme Verlag KG

Stuttgart · New York · ISSN 0942-6035 